\title{
Considerations in software character generation
}

\author{
GLEN A. TAYLOR, DAVID KLITZKE, and DOMINIC W. MASSARO \\ University of Wisconsin, Madison, Wisconsin 53706
}

\begin{abstract}
Two concerns about the development of a computer-driven display system to achieve tachistoscopic displays are discussed. Approaches to solving these problems are described.
\end{abstract}

Our tachistoscopic computer display system has been described in detail elsewhere (Taylor, Klitzke, \& Massaro, 1978). In brief summary, the laboratory computer is a DEC LSI-11 with $20 \mathrm{~K}$ words of memory. The display hardware consists of simple display monitors (Tektronix 604) driven through two 12-bit digital-toanalog (D/A) converters to plot the visual displays. The display software consists of three routines. The first routine, the character translator, converts abstract descriptions of a character set into relative $(x, y)$ coordinate pairs. The second routine, the display initializer, converts a user's instructions about which characters he wants displayed and where and how he wants them displayed into a buffer of points that can be presented by the final routine, the display driver.

The development of the display software was guided by two concerns. First, text displays should simulate to the greatest extent possible the appearance of normal printing. Second, the display routines should permit control over the presentation of a display such that any given character can be presented in a precisely known fashion, regardless of the other characters in the display. This paper focuses on these two concerns.

Perhaps the most common solution to composing a character set of discrete points is to use a grid for character definition. For example, characters are often printed on a cathode-ray tube (CRT) screen by illuminating points in a grid seven rows high by five columns wide. Although this approach is very simple and inexpensive, often being built right into the hardware, the resulting characters have several undesirable properties. Small grid systems such as the 7 by 5 dot matrix do not permit adequate approximation of curved and diagonal line segments, resulting in letters with rather poor legibility. Although it can frequently be advantageous to vary character size, this can only be accomplished in one of two ways with a grid system. Either the characters need to be entirely redefined in a larger or smaller grid, a tedious process, or the interpoint spacing of the existing grid cells can be varied.

This work was supported by the National Institute of Education, Department of Health, Education, and Welfare, through its support of the Wisconsin Research and Development Center for Cognitive Learning. Requests for reprints should be addressed to Glen A. Taylor, Wisconsin Research and Development Center, 1025 W. Johnson Street, Madison, Wisconsin 53706.
Grid characters owe much of their legibility to the close proximity of the points. Spreading these points further reduces legibility. The user who has this scheme fixed into hardware has no choice, but if software character generation is used, then the undesirable features of small dot matrices can be avoided.

The practical alternative to the dot matrix approach is to provide an abstract description of the character that a program can translate into the actual points to be plotted. Our implementation permits a character to be constructed from any number of straight-line segments of any orientation and circular arcs of any radius. This scheme has several major advantages. First, the definition of a character set is relatively easy. Although most users will probably be content to utilize a standard character set such as that shown in Figure 1, it is possible to add characters, change characters, or substitute an entirely different character set simply by substituting new definitions. A second advantage is accuracy in approximating the desired letter form. The approximation is constrained only by the resolution of the D/A channels and not by the limited resolution of a much smaller grid system. Thus, legible reproduction of even relatively complex characters such as Chinese ideographs can be accomplished with no more effort than standard English letters. Finally, the size scaling

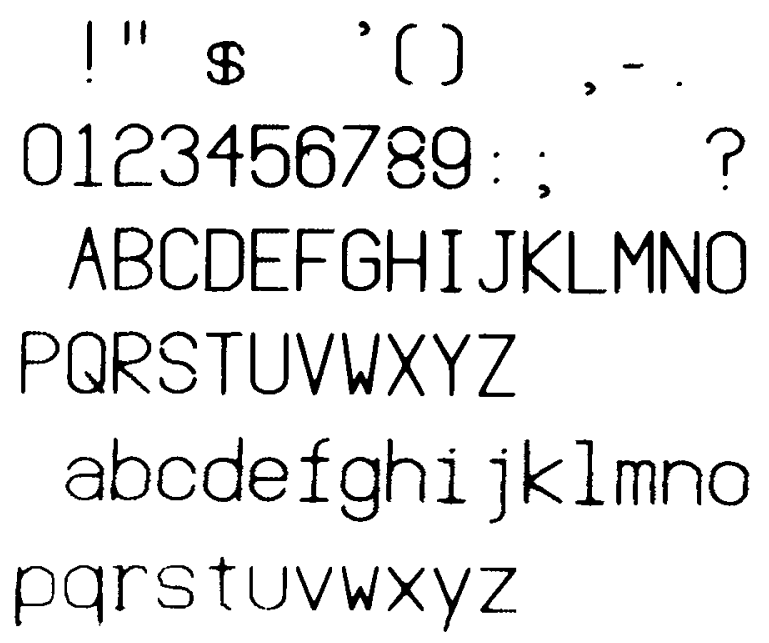

Figure 1. An oscilloscope photograph of the character set presently in use in the authors' laboratory. Before reduction, the capital letters were .25 in. in height. 
and number of points per character may be varied easily, since the letter forms are defined abstractly. The actual number and placement of points in the character are determined by the character-translation routine, with size scaling and point density as user-specified parameters.

In displaying characters on a CRT, a detail that can easily be overlooked is how the characters are actually plotted. Our attention has focused on the implications of two aspects of this process. On nonstorage CRTs, a visual display mist be continually refreshed to remain visible. At refreshment rates high enough to avoid flicker, brightness is a direct function of refreshment rate. If brightness is to be controlled, then the refreshment rate must be precisely controlled. One method is to synchronize refreshment rate with a hardware clock. A second method is to use the plotting routine itself as a software clock. Completion of one refreshment cycle permits immediate commencement of the next refreshment cycle. Such an approach can be effective, but only if the plotting activity consumes the same amount of time from display to display. Assuming that plotting time is a direct function of the number of points to be plotted, this implies that all displays that are to be uniformly bright must contain the same number of points. However, the number of points per character varies, so that even displays with the same number of characters will usually contain slightly different numbers of points. Thus, if plotting time is to be used to control refreshment rate, it is necessary to be able to pad displays with "invisible points" to insure that all displays are equivalent. The introduction of "invisible points," or dead time, into the plotting routine can be accomplished by including some activity that takes the same amount of time as plotting points but that produces nothing visible on the CRT.

In addition to controlling plotting time through the addition of dead time, it is necessary to consider in what order the display should be plotted. Two points of view may be taken. On the one hand, characters may be plotted sequentially in some fixed order such as left to right, top to bottom for lines of text. This approach has the advantage of following Sperling's (1971) advice to plot spatially adjacent points as close together in time as possible to avoid jitter. A second approach is to plot the points in a display in an entirely random order. This approach distributes the luminous energy of the display more evenly during the plotting interval and thus more nearly simulates parallel presentation of the display. Perhaps a choice between these two extremes is of no consequence as long as the total plotting time is suffi- ciently brief. However, regardless of the approach that is chosen, it would be unwise to plot characters differently from display to display. For example, we have chosen to randomize the points to be plotted in a display. If no heed were paid to how this is accomplished, a given character plotted alone would be plotted differently when the character is plotted as part of a multicharacter display. For a single character, randomizing the points does not alter the fact that they will be plotted as a group, whereas randomizing the points of that character along with points from other characters implies that each character will be plotted diffusely during the plotting interval. For this reason, we randomize the points within a character and then divide each character into groups called "bursts." Invisible points and invisible letters are treated in the same manner as visible characters by also being divided into bursts. The order of presentation of bursts within a display is then randomized. This procedure insures that the probability of contiguously plotting any group of points from a single character is constant for all displays with the same total number of characters, visible and invisible.

In summary, we have confined our discussion to two aspects of our tachistoscopic display system that are its most novel features. In so doing, many features of software character generation have been ignored. These have been discussed elsewhere (Gillman \& Buckley, 1973; Taylor et al., 1978). Briefly, however, there is a price for software character generation. One cost is the memory space dedicated to the display software, character set representation, and buffers to accommodate the points being plotted in the active display or displays. Anywhere from hundreds to thousands of words may be required for this purpose. The second major cost is total dedication of the CPU during plotting activity. However, for laboratory environments where these resources can be spared, software character generation provides an extremely flexible and effective means of achieving tachistoscopic displays.

\section{REFERENCES}

Gillman, C. B., \& Buckiey, P. Software character generation. Behavior Research Methods \& Instrumentation, 1973. 5, 228-230.

SPERLING, G. Flicker in computer-generated visual displays: Selecting a CRO phosphor and other problems. Behavior Research Methods \& Instrumentation, 1971, 3, 151-153.

Taylor, G. A., Klitzke, D., \& Massaro, D. W. A visual display system for reading and visual perception research Behavior Research Methods \& Instrumentation, 1978, 10 148-153. 